Ciencia y Salud, Vol. II, No. 1, enero-abril, 2018 •ISSN: 2613-8816

\title{
INTOXICACIÓN CON AMLODIPINA CON HIPOTENSIÓN Y EDEMA PULMONAR NO CARDIOGÉNICO
}

\author{
Intoxication with Amlodipine with hypotension \\ and non-cardiogenic pulmonary edema
}

\section{Dra. Mercedes Ramos*, Dr. Pavel Graciano*, Dra. Doris Cabrera*, Dr. César Mota**, Dra. Lisandra Corona***}

Recibido: 28/8/2017 Aprobado: 25/10/2017

DOI: http://dx.doi.org/10.22206/cysa.2018.v2i1.pp21-24

\begin{abstract}
Resumen
Las intoxicaciones por medicamentos han sido, en los últimos ańos, una importante causa de morbilidad y mortalidad en edades pediátricas. La Amlodipina es un grupo de dihidropiridina de Bloqueadores de Canales de Calcio (BCC) con una vida media de 30-50 horas y con un gran volumen de distribución. En este trabajo describimos una fémina de 14 ańos de edad, que ingiere 20 pastillas antihipertensivas tipo calcio antagonista (Amlodipina). A los tres días, presenta hipotensión y datos clínicos de edema pulmonar. Entonces, se procede a colocar a la paciente en ventilación asistida, proporcionándole infusión de dobutamina, adrenalina y bolus de gluconato de calcio, mejorando así el cuadro clínico en 72 horas.
\end{abstract}

Palabras clave: intoxicación; amlodipina; hipotensión; edema pulmonar.

\footnotetext{
* Residente de Pediatría general. mercedesramos024@gmail

** Fellow Emergencias pediátricas HIRRC

*** Jefa de Emergencias pediátricas del Hospital Infantil Robert Reid Cabral (HIRRC)
}

\begin{abstract}
Drug poisoning in recent years has been an important cause of morbidity and mortality in pediatric ages. Amlodipine is a dihydropyridine group of calcium channel blockers with a half-life of 30-50 hours and a large volume of distribution. In this paper, we describe a 14-year-old female who ingests 20 calcium-blocking antihypertensive tablets (Amlodipine). By the third day, she presented hypotension and clinical data of pulmonary edema. The patient was placed in assisted ventilation with infusion of dobutamine, adrenaline and bolus of calcium gluconate, improving her clinical condition in 72 hours.
\end{abstract}

Keywords: intoxication; amlodipine; hypotension; pulmonary edema.

\section{Introducción}

Las intoxicaciones por medicamentos, drogas, productos domésticos e industriales han sido, en los últimos años, una importante causa de morbilidad y mortalidad en edades pediátricas ${ }^{1}$. Los Bloqueadores de Canales de Calcio (BCC) constituyen la principal categoría de fármacos cardiovasculares implicados en la intoxicación y en un 48\% de las muertes por sobredosis ${ }^{2}$. La Amlodipina es un agente bloqueador del canal de calcio de dihidropiridina utilizado en el tratamiento de la 
hipertensión esencial y la angina de pecho ${ }^{3}$. A diferencia de otros bloqueadores de los canales de calcio, la Amlodipina tiene un aclaramiento metabólico muy bajo, con la ventaja de usar una dosis diaria para mantener una concentración plasmática casi constante ${ }^{4}$.

Tratar a los pacientes que han sufrido una sobredosis de este medicamento puede ser un reto, incluso para el médico más experimentado. La dificultad surge porque los pacientes gravemente intoxicados con BCC pueden tener bradicardia refractaria profunda, hipotensión, lesión renal aguda y edema pulmonar cardiogénico o no cardiogénicos.

\section{Reporte del caso}

Fémina de 14 años de edad que ingiere 20 pastillas antihipertensivas tipo calcio antagonista (Amlodipina). Inicialmente, es evaluada $y$

\section{Figura 1: Radiografía de tórax}

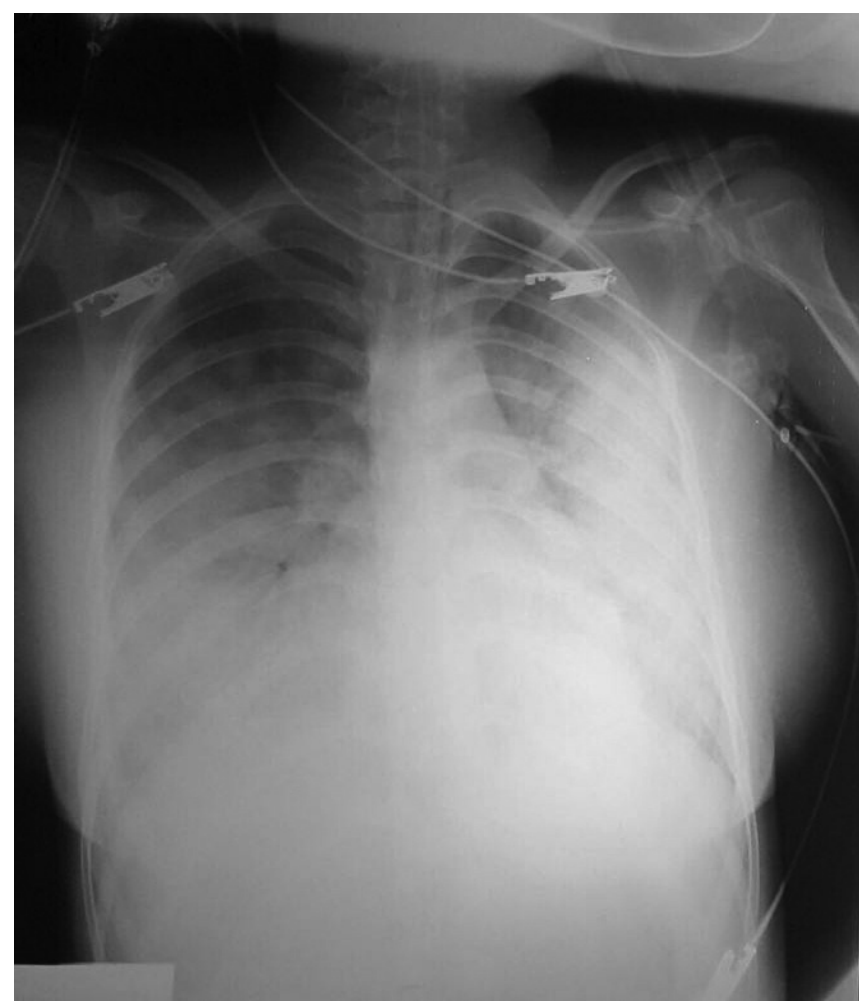

despachada en un centro de atención de primer nivel. A los tres días presenta vómitos, por lo que vuelve al centro de salud donde la encuentran hipotensa; es manejada con líquidos endovenosos y es referida a nuestro centro. Al momento de su llegada, la paciente se presenta irritable, con palidez de piel y mucosa, llenado capilar mayor de 3 segundos, saturando $48 \%$ por oximetría de pulso, frecuencia cardíaca en $110 \mathrm{~L} / \mathrm{m}$ y niveles tensionales de 70/40 mmhg. En el examen físico se ausculta disminución de murmullo vesicular en las bases de ambos pulmones y crepitantes húmedos bilaterales.

Se procede a colocar la paciente en ventilación asistida por tubo endotraqueal y se coloca infusión de Dobutamina y Adrenalina. En sus análisis de laboratorio reporta hemograma con $29 \times 10^{3}$ de glóbulos blancos, $9 \mathrm{~g} / \mathrm{dl}$ de hemoglobina y $368 \times 10^{3}$ de plaquetas, una glicemia capilar en $274 \mathrm{mg} / \mathrm{dl}$ y gases arteriales con PH: 7.23, PCO2: $43.5 \mathrm{mmhg}$ PO2:37.7 mmhg HCO2: 18 y SatO2: 58\%. Las 
pruebas de función renal y electrolitos se encuentran dentro de los límites normales. Se coloca sonda vesical y presenta diuresis inicial de $605 \mathrm{ml}$ en una hora.

La paciente fue admitida en la unidad de cuidados intensivos pediátricos donde se le realiza una radiografía de tórax con hallazgos compatibles de edema pulmonar (ver figura 1). Su presión arterial gradualmente fue mejorando, por tanto, se remueve la ventilación asistida y las aminas vaso activas, al segundo día. Al tercer día, se suspende el inotrópico y es trasladada a una sala clínica. Al sexto día de manejo, se realiza una radiografía control (ver figura 2), donde se observa una importante mejoría. En consecuencia, es egresada.

\section{Discusión}

La Amlodipina es un grupo de dihidropiridina de BCC con una vida media de 30-50 horas y con un gran volumen de distribución. Los pacientes en los que se prevé una intoxicación que va de moderada a grave se deben observar en un entorno controlado durante al menos 12 horas, si se ingiere una formulación de liberación inmediata, y al menos 24 horas cuando se trata de una formulación de liberación sostenida (o Amlodipina), incluso si el paciente está asintomático ${ }^{6}$.

En el caso que nos ocupa, la paciente presentó síntomas a las 38 horas de la ingesta, manifestados por vómitos, malestar general e hipotensión; fue manejada inicialmente con bolus de líquidos endovenosos en un hospital local y al llegar a nuestro centro presentaba datos de edema pulmonar. Cabe destacar que el bloqueo de los canales de calcio de tipo $\mathrm{L}$ en el músculo liso y la actividad depresora del miocardio a niveles tóxicos puede dar lugar a edema pulmonar cardiogénico ${ }^{7}$.

$\mathrm{Al}$ recibir la paciente en estas condiciones, se inició el manejo con inotrópicos y aminas vasoactivas y bolus de gluconato de calcio, mejorando el cuadro clínico en 72 horas. Cabe señalar que las terapias farmacológicas disponibles para la sobredosis de Amlodipina con hipotensión persistente o depresión miocárdica incluyen soporte inotrópico con agentes adrenérgicos, glucagón, infusión intravenosa de calcio y oxigenación de la membrana extracorpórea en choque refractario ${ }^{8}$.

Otra de las medidas terapéuticas es la terapia hiperinsulinémica. Se debe considerar la terapia con hiperinsulinemia euglucémica en pacientes con sobredosis de bloqueadores de los canales de calcio que sean refractarios a la terapia de apoyo. Los suplementos de calcio intravenosos en las formas de gluconato de calcio y cloruro de calcio también resultan beneficiosos, ya que el aumento del calcio extracelular supera el antagonismo competitivo.

\section{Conclusión}

La sobredosis de Amlodipina puede presentarse con hipotensión prolongada y, en casos graves, con edema pulmonar y choque refractario. La resucitación con líquidos debe ser bien juzgada clínicamente y no precipitar edema pulmonar. Es de suma importancia conocer la farmacodinamia y los efectos secundarios de las drogas cuando se sospecha de una intoxicación en los pacientes pediátricos.

\section{Bibliografía}

1. Prado Vizcaíno $\mathrm{Y}$, Vizcaíno Londián $\mathrm{M}$, Abeledo García C, Prado Vizcaíno E, Leiva Peláez O. Intoxicaciones agudas en pediatría. Rev Cubana Pediatr [Internet]. 2011 dic [citado 13 jul 2017]; 83(4): 356-364. Disponible en: http://scielo.sld.cu/scielo.php?script=sci_arttext\&pid=S0034-75312011000400003\&ln$\mathrm{g}=\mathrm{es}$

2. Watson WA, Litovitz TL, Klein-Schwartz W et al. 2003 annual report of the American 
association of poison control centers toxic exposure surveillance system. American Journal of Emergency Medicine. 2004; 22(5): 335-404.

3. Haria M, Wagstaff, AJ. Amlodipine: A reappraisal of its pharmacological properties and therapeutic use in cardiovascular disease. Drugs. 1995; 50(3): 560-586.

4. Abernethy DR, Schwartz JB. Calciumantagonist drugs. The New England Journal of Medicine. 1999; 341(19): 1447-1457

5. Hirachan A, Baral S, Shareef $M$ et al. Amlodipine overdose with hypotension and noncardiogenic pulmonary edema. Nepalese Heart Journal. 2016; 13(1): 27-29.

6. Rietjens S, De Lange D, Donker D, J Meulenbelt J. Practical recommendations for calcium channel antagonist poisoning National Poisons Information Center, University Medical Center Utrecht, the Netherlands, Department of Intensive Care Medicine, University Medical Center Utrecht The Netherlands journal of medicine 2016; 74 (2): $60-66$

7. Siddiqi TA, Hill J, Huckleberry Y et al. Noncardiogenic pulmonary edema and life-threatening shock due to calcium channel blocker overdose and its management: A case report and a clinical review. Respiratory Care. 2014; 59(2): e15-e21.

8. Onge M, Dubé P, Gosselin $S$ et al. Treatment for calcium channel blocker poisoning: A systematic review. Clinical Toxicology. 2014; 52(9): 926-944. 